\title{
Study of shuttle griping and releasing device of weft insertion mechanism of rigid rapier loom
}

\author{
Chenwang Yuan ${ }^{\mathrm{a}}$, Yingjie Niu ${ }^{\mathrm{b}}$, Ge Chen ${ }^{\mathrm{c}}$, Zhengning $\mathrm{Li}^{\mathrm{d}}$ \\ School of Mechanical Engineering, Donghua University, Shanghai 201620, China \\ ayuanchenwang@163.com, babelniu@126.com, cchange@dhu.edu.cn, d1149146@mail.dhu.cn
}

Keywords: weft insertion, shuttle griping and releasing, ADT.

\begin{abstract}
Rigid rapier loom uses rigid rapier to grip the shuttle and do the reciprocating movement to realize weft insertion. To grip and release the shuttle, a complicated mechanism is used. But usually the stability of the mechanism is not good and the installation is complex. According to simulation and experiment, the following two mechanisms based on different principles are proposed to realize griping and releasing the shuttle. The Axiomatic Design Theory is used to analyses the two schemes and compares their rationality by their design matrixes. Finally, the two schemes' machinability, installation convenience and performance stability are discussed to better understand their difference.
\end{abstract}

\section{Introduction}

Weft insertion is to insert the weft into the shed formed by warps, so that the weft and the warp could form textiles. Weft yarn insertion mechanism is the key mechanism for three dimensional rapier loom. The efficient and quality of the three dimensional fabrics made by the rapier loom depends on the velocity and the rate of success of the weft insertion mechanism greatly [1]. Because of the multiple sheds formed when weaving three dimensional fabrics, the weft insertion rapier should be a rigid rapier with a mechanism that could grip the shuttle and do the reciprocating movement through the sheds with the warp [2].

To grip and release the shuttle, usually a complicated mechanism is used [3]. But usually the stability of the mechanism is not good and the installation is complex. According to simulation and experiment, the following two mechanisms are proposed to realize griping and releasing the shuttle.

\section{Two types of mechanisms}

\subsection{Type of griping by friction (TGF)}

The type of griping by friction mechanism of the rigid rapier loom is as shown in the Figure2-1. TGF mechanism includes 4-rapier, supporting part, shuttle part, 3-clamping sleeve, 2-shuttle box, 1-air cylinder.

The supporting part includes 5-removable holder, 6-immovable holder, the connection between 4-rapier and 5-removable holder is threaded connection. The 4-rapier could slide on the 6-immovable holder. The shuttle part includes 7-shuttle body and 8-friction stick. Two bolts are used to connect the 7-shutttle body and the 8-friction stick. The 3-clamping sleeve is fixed on the 4-rapier by thread. The 1-air cylinder is fixed on the 2-shuttle box by four bolts.

This TGF mechanism of the weft insertion of rigid rapier loom clamps the shuttle part by static friction force between 8-friction stick and 3-clamping sleeve. To detain the shuttle in the shuttle box, aerate the 1-air cylinder after the shuttle enters into the shuttle box to protrude the rod. After the rod is protruded, it would make the 8-friction stick stuck in the shuttle box. Because of the pressure made by the rod of the air cylinder, the static friction force between the 8-friction stick and the 2-shuttle box is much bigger than the static friction force between the 8-friction stick and the 3-clamping sleeve. So the shuttle stays in the 8-shuttle box. To make the shuttle move with the 4-rapier, stop aerating the 1-air cylinder to retract the rod and the pressure on the 8-friction stick caused by the rod would disappear. So that the static friction force between the 8-friction stick and 
the 2-shuttle box is smaller than the static friction force between the 8-friction stick and the 3-clamping sleeve. In this case, the shuttle would move with the 4-rapier to back to the initial position.

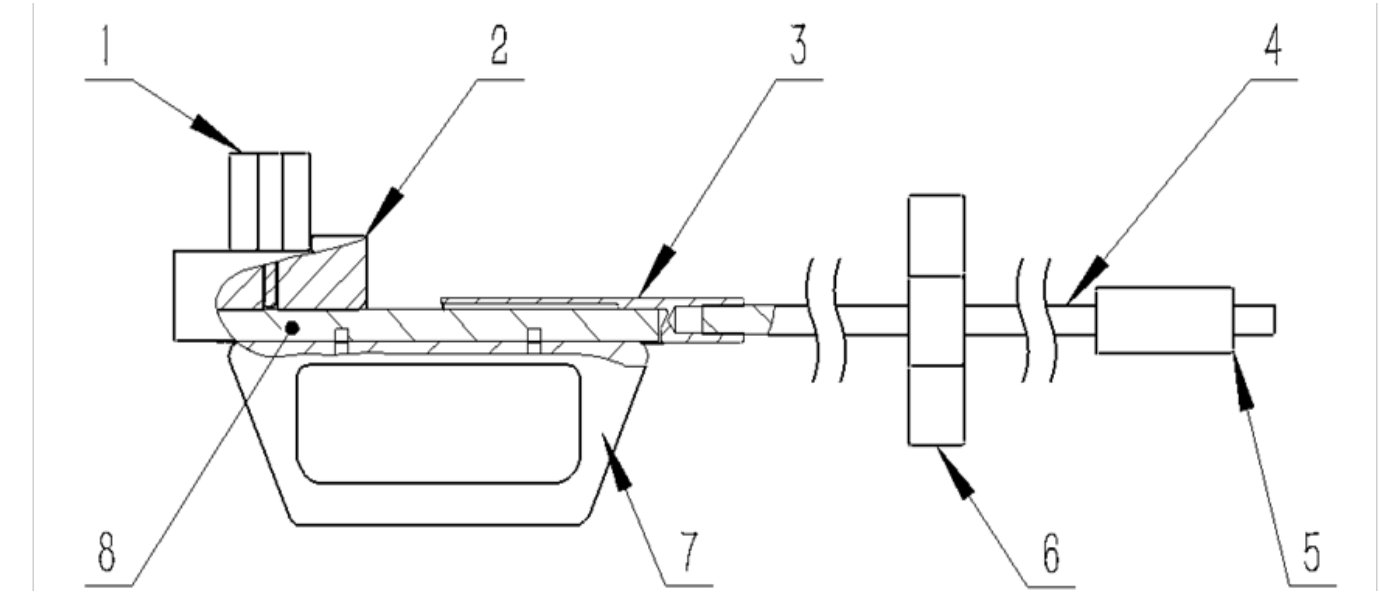

Fig. 2-1 Type of griping by friction mechanism

1-air cylinder 2-shuttle box 3-clamping sleeve 4-rapier 5-removable holder 6-immovable holder 7-shuttle body 8 -friction stick

\subsection{Type of griping by magnetism (TGM)}

The TGF mechanism is easily manufactured, but its reliability is not very high. So the TGM mechanism is proposed for the weft insertion of the rigid rapier loom as shown in the Figure4-2. It includes 5-rapier, the supporting part, the shuttle part, the shuttle griping part, the 2-shuttle box and the 1-air cylinder.

The supporting part is the same as the part in the TGF mechanism. The shuttle part includes 10-shuttle body, 11-adjustment stick, 3-connection body and 4-magnet. The 10-shuttle body, 11-adjustment stick and 3-connection body are connected with bolts. The 4-magnet is fixed on the 3-connection body by thread. The shuttle griping part contains 8- clamping sleeve and 9-magnet. Threads are used to connect 8-clamping sleeve and 5-rapier, 8-clamping sleeve and 9-magnet. The 1-air cylinder is fixed on the 2-shuttle box with bolts.

In this mechanism, 4-magnet and 9-magnet use $\mathrm{Nd}_{2} \mathrm{Fe}_{14} \mathrm{~B}$ material with the brand of $\mathrm{N} 35$ or bigger. The configuration should be $25 \mathrm{~mm}$-diametrical cylinder with a $6 \mathrm{~mm}$-diametrical counterbore.

Aerate the 1- air cylinder after the shuttle enters into the 2-shuttle box and the rod would be pushed out and insert into the groove on the 3-connection body. In this case, the 3-connection body is stuck in the 2-shuttle box and couldn't be dragged out of the 2-shuttle box by the magnetic force between the two magnets. So the shuttle part is separated from the shuttle griping part and stays in the 2-shuttle box. Once stop aerating the 1-air cylinder, the rod would be retracted from the groove on the 3-connection body and the shuttle part could be dragged out of the 2-shuttle box to move with the shuttle griping part. 


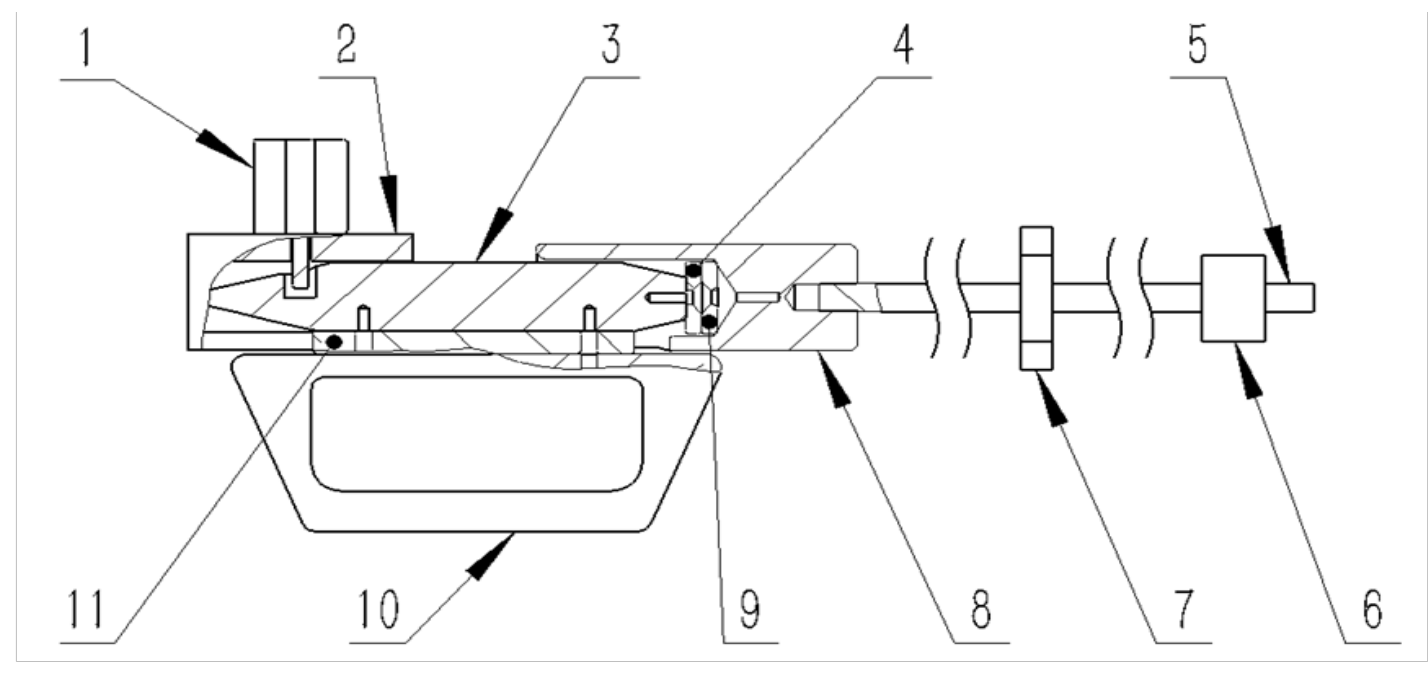

Fig. 4-2 Type of griping by magnetism mechanism 1-air cylinder 2-shuttle box 3-connection body 4-magnet 5-rapier 6-removable holder 7-immovable rapier 8-clamping sleeve 9-magnet 10-shuttle body 11-adjustment stick

\section{Principle analysis based on Axiomatic Design Theory}

\subsection{Axiomatic design theory (ADT)}

Axiomatic design theory is a system design methodology to systematically analyze the customers' needs and the design solutions [4]. It is a theory primarily based on two axioms and they are supposed to guide into a design that is sound. The ADT was developed around the 80s and 90s at MIT led by Num P. Suh. Since then, there have been many developments with ADT in literature, including guidelines for applying ADT to practical design or decision-making problems.

ADT defines the customers' needs in functional requirements (FRs), and defines the design solutions as design parameters (DPs) that are supposed to fulfill FRs. The design process can be viewed as a mapping from the functional requirements domain to the design parameters domain. The mapping can be represented as the following formula.

$$
\{\text { FRs }\}=[A]\{\text { DPs }\}
$$

In the formula (3-1), [A] is the design matrix based on the FRs and DPs and the design process is guided by $[\mathrm{A}]$. ADT maintains the independence of the FRs, which suggests that all FRs should be uncoupled or decoupled.

\subsection{Functional Requirements and Design Parameters}

Functional requirements (FRs) of the shuttle griping and releasing device are classified based on the action. The shuttle griping and releasing device is used to grip, hold and release the shuttle once the time is right. So the FRs derived from the general architecture of the device are given as follows:

FR1: To enable the device to grip the shuttle.

FR2: To enable the device to hold the shuttle.

FR3: To enable the device to release the shuttle.

According to the FRs, the two schemes have different ways to meet the requirements. As the two schemes both use interaction force to achieve the goal, DPs are displayed in couples.

For the first scheme, the friction force between the 3-clamping sleeve and the 8-friction stick enables the device the grip the shuttle. So the 3-clamping sleeve and the 8-friction stick are the key to meet the FR1. After griping the shuttle, the 3-clamping sleeve and the 8-friction stick are also responsible for holding the shuttle, which is the FR2. To meet the FR3 to release the shuttle, the friction between the 8-friction stick and the 2-shuttle box is used.

DP11: Part 3 and 8 
DP12: Part 3 and 8

DP13: Part 2 and 8

For the second scheme, the magnetic force between the 9-magnet on the 8-clamping sleeve and the 4-magnet on the 3-connection body enables the device to grip the shuttle and the 8-clamping sleeve and the 3-connection body are used to make the shuttle be held during movement. To release the shuttle, the rod of the 1-air cylinder makes the 3-connection body stuck in the 2-shuttle box.

\section{DP21: Part 4 and 9 \\ DP22: Part 3 and 8 \\ DP23: Part 2 and 3}

\subsection{Design Equation}

According to the ADT, the relationship between FRs and DPs can be represented by the following design equation:

For schene1:

$$
\left\{\begin{array}{l}
\text { FR1 } \\
\text { FR2 } \\
\text { FR3 }
\end{array}\right\}=\left[\begin{array}{ccc}
\times & \times & \mathrm{O} \\
\times & \times & \mathrm{O} \\
\mathrm{O} & \mathrm{O} & \times
\end{array}\right\}\left\{\begin{array}{l}
\mathrm{DP} 11 \\
\mathrm{DP} 12 \\
\mathrm{DP} 13
\end{array}\right\}
$$

For schene2:

$$
\left\{\begin{array}{l}
\text { FR1 } \\
\text { FR2 } \\
\text { FR3 }
\end{array}\right\}=\left[\begin{array}{ccc}
\times & \mathrm{O} & \mathrm{O} \\
\mathrm{O} & \times & \mathrm{O} \\
\mathrm{O} & \mathrm{O} & \times
\end{array}\right\}\left\{\begin{array}{l}
\mathrm{DP} 21 \\
\mathrm{DP} 22 \\
\mathrm{DP} 23
\end{array}\right\}
$$

According to the equation (3-2) and (3-3), it can be seen that the design matrix of scheme 1 is coupled as the part 3 and 8 have to meet not only the first function requirement, but also the second function requirement. That would make the FR1 and FR2 interact each other. But the design matrix of scheme 2 satisfies the theory of ADT as it is uncoupled. Ensure the design matrix uncouple could make the mechanism work stably.

According to the analyses above, the performance of scheme 2 would be more stable than the scheme 1.

\section{Scheme comparison}

\subsection{Machinability}

The two schemes share some parts including removable holder, immovable holder, rapier, air cylinder, shuttle box and shuttle body. For removable holder and immovable holder, aluminium could be used to reduce the weight. When it comes to the machining, a common milling machine is qualified. The rapier uses $10 \mathrm{~mm}$ - diametrical hardened and tempered steel bar. The threads could be processed on the turning lathe. The air cylinder could be purchased. The processing of the shuttle box is a little bit difficult because it needs a formed cutter to mill out the groove. The rest surfaces are not difficult to process. The shuttle body should be customization.

The differences between two schemes are the shuttle part and the shuttle griping part. For the first scheme, the clamping sleeve and the friction stick are the peculiar parts. Quenched and tempered aluminum is used to process the clamping sleeve to reduce the weight and formed cutter is used to produce the trapezoidal groove which should fit with the friction stick. The clamping sleeve also needs a $10 \mathrm{~mm}$-diametrical thread at the bottom. The friction stick uses nylon as its material for it is easy to process and punch on the nylon.

For the second scheme, the clamping sleeve, connection body, magnet and adjustment stick are the peculiar parts. The clamping sleeve is a revolved body which could be processed on the turning lathe. The processing is difficult for that it is a thin-walled part and needs an axial slot which makes it difficult to be clamped on the milling machine. The processing of the connection body is similar to the clamping sleeve. The turning lathe is used to process the outer profile and the milling machine is used to process the slot. A threaded hole is also needed at the bottom of the clamping 
sleeve. To get a lower weight and a higher machinability, aluminum is chosen as the material. The adjustment stick has two through-holes on it, not as the friction stick.

Based on the analyses above, the machinability of the scheme of TGF is better than the scheme of TGM. The scheme of TGF has fewer parts and they are easier to process.

\subsection{Installation convenience}

In order to discuss the installation convenience, we need to figure out the theoretic differences between two schemes. According to Scheme 1, the shuttle is gripped by the friction force between the 3-clamping sleeve and the 8-friction stick. And it's separated on account of the friction force between the 8-friction stick and the inside of the 2-shuttle box being greater than the friction force between the 8-friction stick and the 3-clamping sleeve. As for Scheme 2, the shuttle is gripped by the powerful magnetic force between the 9-magnet on the 8-clamping sleeve and the 4-magnet on the 3-connection body. While cylinder rod being ejected to jam into the slot of the head of the 3-connection body, the shuttle is separated from clamping sleeve.

With regard to Scheme 1 , that the friction force being the key to control the griping and separating makes the motion stability be susceptible to the control of friction force. Under the circumstance of friction coefficient being constant, control of friction force equals to control of interaction force, which is hard to control during the installation, taking dimensions and tolerance, material deformation and installation error into consideration. Friction force between the 8-friction stick and the inside of the 2-shuttle box and the friction force between the 8-friction stick and the 3-clamping sleeve interacts with each other, both of which are hard to control. Also, the former is a highly possible to be greater than the latter, causing the shuttle to be unable to be pulled out after getting into the shuttle box even when the cylinder rod isn't ejected. Therefore, the Scheme 1 demands high installation accuracy and is poor in installation convenient, and expected effect won't appear before massive debugging.

As for Scheme 2, that powerful magnetic force between two permanent magnets being the key to control the griping and separating demands not that high installation accuracy. Only if the design demand is met, permanent magnets on the 8-clamping sleeve and the 3-connection body contact with each other, the magnetic force will be powerful enough to make the shuttle and the rapier move together using a diameter of $25 \mathrm{~mm}$, brand of $\mathrm{N} 35 \mathrm{Nd}_{2} \mathrm{Fe}_{14} \mathrm{~B}$. When the cylinder rod is ejected to jam into the slot of 3-connection body, the 3-connection body with the shuttle will be separated with the 8-clamping sleeve and stay in the 2-shuttle box. Thus, the Scheme 2 is much more convenient and doesn't need high installation accuracy.

\subsection{Performance stability}

Performance stability of the mechanism mainly depends on the stability of griping and releasing the shuttle under the situation of long working hours, during which the stability of griping and releasing the shuttle is influenced by the wear of the key parts.

With regards to Scheme 1, wear would definitely occur according to the character of friction force, decreasing the friction force and making it unstable to gripe and release. Once shuttle fails to leave with the rapier after getting into the shuttle box, shuttle may even be broken down by the weft yarn insertion mechanism.

As for Scheme 2, powerful magnetic force will still exist and stay stable even after long hours of working and the release of shuttle depends on cylinder rod's jam into the slot on the connector. Failures won't happen due to the better stability.

\section{Conclusion}

From the analyses and discussion above, the two schemes have their own advantages and disadvantages. From the Table 1, conclusions can be made that the type of griping by magnetism mechanism is better for weft insertion because, as for machines, the most important thing is its performance stability. 
Table 1 Comparation of TGF and TGM

\begin{tabular}{|l|c|c|}
\hline Scheme & TGF & TGM \\
\hline Griping force & Static friction force & Magnetic force \\
\hline Separating way & Static friction force & Slot \\
\hline machinability & Good & Poor \\
\hline Installation convenience & Poor & Good \\
\hline Performance stability & Poor & Good \\
\hline
\end{tabular}

\section{Reference}

[1] Xiong Zhao, Bing Xu, Jianneng Chen, et al. "Several basic weft insertion mechanisms with innovation changes of rapier loom”. Textile Machinery, 2008 (2), p. 48 51.

[2] Siwei Zhao, Yong He, Ming Chen, et al. “A new way for weft insertion motion on rapier weaving machine”. Shanghai Textile Science \& Technology. Vol. 33 (2005) No. 5, 2005. p. 26-28.

[3] Zhichao Zhu, Zhihe Fang, "Optimization design of a weft insertion mechanism for rapier looms”, Journal of Donghua University (English Edition), Vol. 20 (2003) No. 3, p. 38-41.

[4] Sun, N. P., et la. “The Principle of Design”, Oxford, New York: Oxford University Press, 1990. 